\title{
FROM THE DIRECT NUMERICAL SIMULATION TO SYSTEM CODES - PERSPECTIVE FOR THE MULTI-SCALE ANALYSIS OF LWR THERMALHYDRAULICS
}

\author{
D. BESTION \\ CEA-Grenoble, DEN-DER-SSTH, 17 rue des Martyrs \\ 38054, GRENOBLE, FRANCE \\ ${ }^{*}$ Corresponding author. E-mail : dominique.bestion@cea.fr
}

Received November 30, 2010

A multi-scale analysis of water-cooled reactor thermalhydraulics can be used to take advantage of increased computer power and improved simulation tools, including Direct Numerical Simulation (DNS), Computational Fluid Dynamics (CFD) (in both open and porous mediums), and system thermalhydraulic codes. This paper presents a general strategy for this procedure for various thermalhydraulic scales. A short state of the art is given for each scale, and the role of the scale in the overall multi-scale analysis process is defined. System thermalhydraulic codes will remain a privileged tool for many investigations related to safety. CFD in porous medium is already being frequently used for core thermalhydraulics, either in 3D modules of system codes or in component codes. CFD in open medium allows zooming on some reactor components in specific situations, and may be coupled to the system and component scales. Various modeling approaches exist in the domain from DNS to CFD which may be used to improve the understanding of flow processes, and as a basis for developing more physically based models for macroscopic tools. A few examples are given to illustrate the multi-scale approach. Perspectives for the future are drawn from the present state of the art and directions for future research and development are given.

KEYWORDS : Thermalhydraulics, System Code, CFD, Nuclear Reactor, Multi-Scale Analysis

\section{INTRODUCTION}

The current generation of thermalhydraulic $(\mathrm{TH})$ system codes (such as CATHARE [1, 2], RELAP 5 [3], TRACE [4], ATHLET [5]), are widely used for nuclear reactor simulation in normal operation, and in accidental transients. These codes were designed in the 1970s and reached their maturity after two or three decades. During the past 15 years, there have been many opportunities to establish the state of the art of these system codes. At the end of the nineties OECD/CSNI organized several Workshops on Transient Thermal-Hydraulic and Neutronic Codes (Aix en Provence, 1992 [6], Annapolis, 1996 [7], Barcelona, 2000 [8]). The French FASTNET project evaluated the capabilities and limitations of system codes [9], and these reflections were later extended by a community of European experts in the EUROFASTNET [10] project. The objective of these projects was to apprise the state of the art of the current methods, including the physical and numerical models implemented in current TH codes, and the experimental methods. This state of the art, along with a review of industrial needs for the medium term, allowed experts to identify various ways of progress.

All of these system codes underwent an extended period of development, validation, and verification. The underlying modeling and numerical methods benefited significantly from the extensive nuclear reactor safety programs which were carried out primarily in the 70 s and ' 80 s.

Despite some shortcomings and limitations, all of the system codes have attained a high level of maturity, as indicated by their principal capabilities both qualitatively and, in many cases, quantitatively, over a wide spectrum of thermalhydraulic conditions in LWR accident scenarios. However, this improvement has often been the result of a "learning" process involving how to make an optimal choice of code models, code options, and numerical and/or nodalization details. This process is acceptable as long as sufficient experimental data exist to allow the quality of the predictions to be accurately judged. After 30 years of validation and improvements, system codes can predict the main phenomena of most accidental transients of PWR \& BWR with reasonable accuracy and 
produce reliable conclusions on safety issues.

Current system codes use the two-fluid model with algebraic empirical formulations of constitutive laws describing interfacial and wall-to-fluid transfer processes. The acknowledged limitations of these codes include a high degree of empiricism and a lack of dynamical modeling of the interfacial area and flow regime transitions. In 3D models, other limitations include the lack of adequate turbulence modeling and the use of idealized friction tensors in rod bundles. Both 1-D and 3-D models have limited capabilities for flow regime, where a phase is present under the form of separate fields having different dynamic behaviors, such as a liquid film and a droplet field. Further progress may be obtained by additional equations (such as transport equations for interfacial area or for turbulent scales), multi-field modeling, or better spatial and temporal resolutions of 3D calculations. It was eventually determined that a new generation of codes would be necessary to allow for the best implementation of the new models, including coupling between microscopic and macroscopic models, and coupling of thermal-hydraulics to neutron kinetics, fuel thermo-mechanics, and structure mechanics. These conclusions were at the origin of the NEPTUNE multiscale thermalhydraulic platform developed and financed by CEA, EDF, AREVA and IRSN in France [11, 12]. A similar multi-scale TH platform was also developed in Europe in the NURESIM [13] and NURISP [14] projects.

In accordance with these reflections, single-phase CFD tools were increasingly applied to investigate and simulate some reactor issues related to turbulent mixing, such as boron mixing, hot and cold water mixing related to Pressurized Thermal Shock (PTS) or Steam Line Break (SLB), and containment thermalhydraulics. At the end of the 1990s, the CFD codes were extended to two-phase flow and several Interface Tracking Methods (ITM) were developed, including Volume of Fluids (VOF), Front Tracking (FT), Level set (LS) and others. This appearance of myriad new TH simulation scales, at the end of the extensive experimental programs, opened a new way towards the two-phase Direct Numerical Simulation (DNS) and put forward the concept of "multi-scale analysis". In this concept, small scale simulation somewhat compensates for the lack of new experimental data.

This paper presents how this "multi-scale analysis" of water-cooled reactors can be used to take advantage of increasing computer power and improved simulation tools. The role of DNS, CFD in open medium, CFD in porous medium, and system TH codes is defined. In this process, system TH codes will maintain an important role and CFD in porous medium will be increasingly used with a refined space resolution. CFD in open medium allows zooming on some reactor components in certain situations, and may be coupled to the system and component scales. Various modeling approaches exist in the domain from DNS to CFD which may be used to improve the understanding of flow processes and as a basis for developing more physically based models for macroscopic tools. A few examples are given to illustrate the multi-scale approach. Perspectives for future are drawn from the present state of the art and directions for future research and development are given.

\section{THE MULTI-SCALE ANALYSIS OF REACTOR THERMALHYDRAULICS}

The multi-scale analysis of reactor thermalhydraulics is often illustrated with four scales corresponding to four kinds of simulation software, as shown in Figure 1. The four scales are the system scale, the component scale, the meso-scale (also referred to as CFD scale), and the microscale (also referred to as DNS scale).

The system scale is dedicated to the overall description of the circuits of the reactor. The primary and secondary circuits of a reactor are modeled by coupling $0 \mathrm{D}, 1 \mathrm{D}$, and 3D modules together with sub-modules for pumps, valves, breaks, safety systems, heat exchangers and control systems. Pipes are treated by a 1D model, pipe connections with Tees, large volumes with 0-D modules, and the pressure vessel may be modeled with a 3D module using CFD for porous medium. The whole reactor is modeled using a few hundred OD and 1D meshes, whereas a 3D pressure vessel modeling currently uses about $10^{3}$ coarse meshes. This method allows simulations of all accident scenarios, including Large Break Loss of Coolant Accidents (LBLOCA) and Small Break LOCAs (SBLOCAs), with a reasonable CPU time (less than 12 hours of a singleprocessor computer).

The component scale also uses CFD in porous medium. This scale is dedicated to the design, safety, and operation studies of reactor cores and tubular heat exchangers (steam generators, condensers, auxiliary exchangers). Rod or tube bundles are homogenized into the control volumes using the "porosity" concept. The minimum spatial resolution is fixed by the sub-channel size (scale centimeter), which gives the sub-channel analysis.

The meso-scale requires a CFD in open medium. The average scale (one millimeter or less) goes beyond the limits of the component scale for a finer description of the flows. This scale includes turbulence modeling using the RANS or URANS approach, and envisages new approaches similar to the LES, as well as local analysis of the critical parts of the cores, steam generators, or other components, including those with complex geometries. This is also the only scale able to predict the fluid temperature field for investigating thermal shocks or thermal fatigue of the reactor structures.

The micro-scale corresponds to the DNS or pseudoDNS approaches and may also include some LES-like approaches with ITM. The characteristic length may be one micrometer or less, allowing for local simulations 
that focus on very small domains (e.g. containing a few bubbles or droplets).

Since the boundaries of the four scales are not entirely clear, it is necessary to classify the various modeling approaches [15, 16], as summarized below.

Figure 1 also shows some connections between the four scales, but the nature of these links has yet to be defined. A coupling of several scales may be used to solve a thermalhydraulic issue, but it may also be possible to apply a multi-scale analysis method without any coupling.

\subsection{Multi-Scale Coupling and Multi-Scale Analysis}

Coupling between two or more scales may be used when a zoom on a specific part of the simulation domain is necessary, as when there is a particular interest in small scale phenomena taking place in a limited part of the domain. As illustrated in Figure 1, it is possible to conceive of a system code which can predict the behavior of the primary circuit which gives boundary conditions to a component code for the core thermalhydraulics. Within the core, a few sub-channels could be simulated with a CFD for open medium, using the component code results as boundary conditions. Finally, a DNS of a very small part of a sub-channel may be used to predict a very local phenomenon, such as a Departure from Nucleate Boiling (DNB) occurrence. It may one day even be possible to create an ideal simulation tool with an automatic refinement of the model resolution, depending on the phenomenon to be captured.

But this goal is still a long way off, and there are many difficult challenges impeding rapid progress in this area. Coupling two scales induces three kinds of problems:

1. The code architecture should be adapted to allow for an easy transfer of data from one solver to the other, and a supervisor must manage the work schedule for each module.

2. The numerical schemes used in both solvers have to be made compatible with each other and the numerical coupling should not degrade the solution

3. The physical coupling of two models with different space and time resolutions creates many issues, such as:

- How to create inlet boundary conditions for a finer model from a coarser model?

- What is the impact of using coarse boundary conditions for the fine model simulation? What space and time domain is necessary to lose the memory of these degraded boundary conditions? How far upstream of the domain of interest one should locate the coupling surface to get a non-degraded solution in the domain of interest?

- What physical quantities have to be preserved at inlet and outlet boundary conditions (mass conservation, energy conservation, turbulence...)?

In the present state of the art, the first type of problem has been addressed for some couplings of system code with component code, or coupling of system code with CFD code. R\&D is in progress for the second and third types of problems. However, many issues related to the numerical and physical aspects of the coupling remain to be addressed, particularly in two-phase conditions.

A more pragmatic view of the multi-scale analysis consists in using the smaller scale simulation without coupling to macroscopic scales. The small scale simulation is used to understand the basic phenomena and to develop more physically based models or closure laws for a more macroscopic model. In the example above of a DNB occurrence in a reactor core, the role of the various scales could be defined as follows:

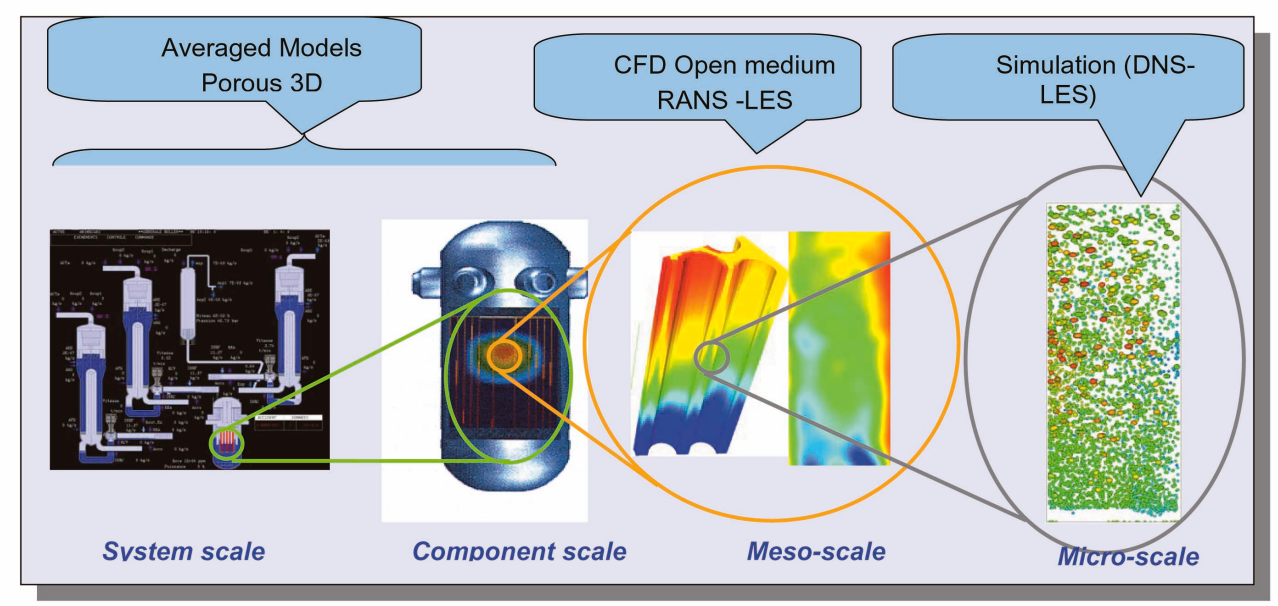

Fig. 1. Illustration of the Multi-Scale Analysis of Reactor Thermalhydraulics 
- DNS or pseudo-DNS simulations may be used to identify the physics of the DNB process and to derive a physically based local DNB criterion for a twophase CFD for open medium using a RANS approach.

- The CFD for open medium using a RANS approach may simulate the few sub-channels which are likely to create conditions for a DNB occurrence (for example, see how this approach is developed in the NEPTUNE,

- A sub-channel analysis code may be used to give boundary conditions to the CFD code.

- A system code may be used to generate core boundary conditions when a DNB may be anticipated, such as a deviation from nominal condition or a Reactivity Insertion Accident (RIA).

\section{THE VARIOUS SCALES OF SINGLE-PHASE AND TWO-PHASE THERMALHYDRAULICS}

\subsection{Classification of the Modeling Scales}

Figure 2 illustrates a classification of the various modeling approaches for reactor thermalhydraulics in two-phase flow. As proposed in [16], the classification is based on successive steps to derive the system of equations used in each approach from basic local instantaneous equations (continuity equation, Navier-Stokes equation, and energy equation). The selection of the respective approaches can be made by considering five successive choices:

1. Selection between the CFD for open medium and the CFD for porous body by multiplying basic equations by a fluid-solid characteristic function
2. Choice of the number of phases or fields of the model by multiplying basic equations by phase characteristic functions or field characteristic functions

3. Time averaging or ensemble averaging

4. Space averaging, space integration, or space filtering

5. Treatment of interface, which can be Deterministic Interface (DI), Filtered Interface (FI) or Statistical Interface (SI)

This classification identifies nine different modeling approaches in two-phase flow and only seven in singlephase flow.

For the porous medium CFD, equations are multiplied by the fluid/solid characteristic function $\chi_{\mathrm{f}}(\mathrm{x}, \mathrm{t})$ :

$\chi_{\mathrm{f}}(\mathrm{x}, \mathrm{t})=1$ when point $\mathrm{x}$ is in the fluid at time $\mathrm{t}$ $\chi_{\mathrm{f}}(\mathrm{x}, \mathrm{t})=0$ when point $\mathrm{x}$ is in the solid at time $\mathrm{t}$ A volume average of $\chi_{\mathrm{f}}$ is the porosity factor:

$$
\phi \cong<\chi_{f}>=\frac{V_{f}}{V}
$$

After multiplication by $\chi_{\mathrm{f}}$, equations are averaged over time and then over a fluid volume, as follows:

$$
<A>_{f}(x, t) \cong \frac{<\chi_{f} A>}{<\chi_{f}>}=\frac{1}{V_{f}(x)} \int_{V(x)} A \chi_{f} d V
$$

The way to introduce the number of fluids or fields is to consider that local instantaneous equations are multiplied by fluid/field characteristic function before any averaging or filtering.

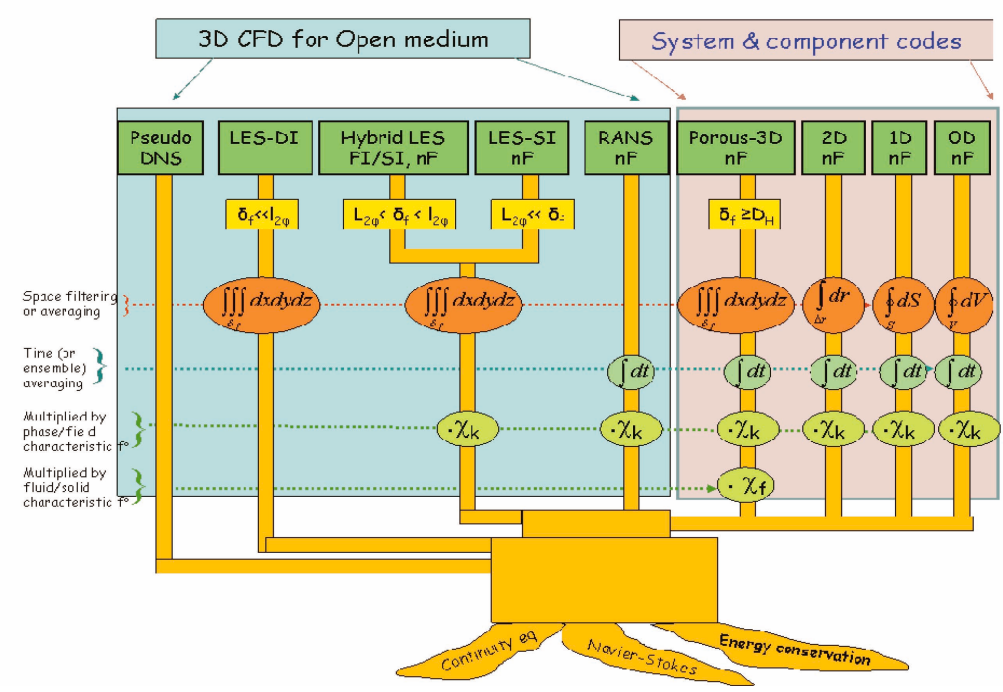

Fig. 2. The Tree of Two-Phase Thermalhydraulic Modeling Approaches 
Let $\chi_{\mathrm{k}}(\mathrm{x}, \mathrm{t})$ be the fluid characteristic function for phase $\mathrm{k}$ or field $\mathrm{k}(\mathrm{k}=1, \mathrm{n})$

$\chi_{k}(x, t)=1$ when point $\mathrm{x}$ is in the phase $\mathrm{k}$ or field $\mathrm{k}$ at time $\mathrm{t}$

$\chi_{k}(x, t)=0$ when point $x$ is not in the phase $\mathrm{k}$ or field $\mathrm{k}$ at time $\mathrm{t}$

One can multiply also by the product $\chi_{k} \cdot \chi_{\mathrm{f}}$ for a multi-field model in a porous body approach. The three basic equations are first multiplied by $\chi_{\mathrm{f}}$ or $\chi_{\mathrm{k}} \cdot \chi_{\mathrm{f}}$ for $\mathrm{k}=1, \mathrm{n}$. Then they are averaged resulting in $3 \mathrm{n}$ averaged balance equations (mass, momentum, and energy).

Time averaging or ensemble averaging is a common way to derive equations for either the RANS (Reynolds Average Navier-Stokes) approach or URANS, when unsteady flow is simulated with a time filter scale.

Space averaging or filtering is also used in the Large Eddy Simulation (LES) of turbulent flows in an open medium context. Space averaging in two-phase flow filters both the small eddies and the interfaces, because the density discontinuity is smeared or diffused, which may cause the shape of the interface to be degraded. In Figure $2, \delta_{\mathrm{f}}$ represents the size of the space filter. $\mathrm{L}_{2 \varphi}$ and $1_{2 \varphi}$ correspond respectively to the largest and smallest interface scale, i.e. the largest and smallest bubble or drop size or interface wavelength.

An interface is "simulated" and is said to be "deterministically treated" when its space and time position is simulated or actually predicted without any simplification. This is clearly the case of a DNS or pseudoDNS where neither space nor time averaging is used.

An interface is "statistically treated" when an averaging or filtering procedure does not allow for the prediction of its space and time position. In such case, only statistical or averaged information on several interfaces may be predicted through quantities such as a void fraction or an interfacial area density. Such a statistical treatment may result from time averaging or from space averaging.

An interface is considered as a "Filtered Interface" when its space and time position is predicted with some filtering of the smaller scale deformations. This filtering may result either from space filter or from time averaging.

When $\delta_{\mathrm{f}} \ll 1_{2 \varphi}$ all interfaces can be simulated

When $\delta_{\mathrm{f}}>\mathrm{L}_{2 \varphi}$ all interfaces are statistically treated

When $1_{2 \varphi}<\delta_{\mathrm{f}}<\mathrm{L}_{2 \varphi}$ some interfaces can be filtered, the smallest being statistically treated

Table 1 summarizes the time and space resolution in the various modeling approaches of single-phase and two-phase CFD. The main difference between single-phase

Table 1. Time and Space Resolution in the Various Modeling Approaches of Single-Phase and Two-Phase CFD

TIME AND SPACE RESOLUTION IN SINGLE-PHASE AND TWO-PHASE CFD

\begin{tabular}{|c|c|c|c|c|c|c|}
\hline & \multicolumn{5}{|c|}{ Open medium } & Porous medium \\
\hline $\begin{array}{l}\text { Time \& space } \\
\text { filtering }\end{array}$ & $\begin{array}{c}\text { No filter } \\
\text { No } \\
\text { averaging }\end{array}$ & \multicolumn{3}{|c|}{ Space filtering } & Time averaging & $\begin{array}{l}\text { Time averaging } \\
\text { Space filtering }\end{array}$ \\
\hline Turbulence & DNS & \multicolumn{3}{|c|}{ LES - VLES } & RANS - URANS & RANS - URANS \\
\hline Single-phase models & DNS & \multicolumn{3}{|c|}{ LES - VLES } & RANS - URANS & Porous medium approach \\
\hline Turbulence & DNS & LES & LES - VLES & LES - VLES & RANS - URANS & RANS - URANS \\
\hline $\begin{array}{c}\text { Interfaces } \\
\text { Simulated } \\
\text { Filtered } \\
\text { Statistical }\end{array}$ & $\begin{array}{l}\text { Simulated } \\
\text { Interfaces }\end{array}$ & $\begin{array}{l}\text { Simulated } \\
\text { Interfaces }\end{array}$ & $\begin{array}{l}\text { Filtered \& } \\
\text { Statistical } \\
\text { Interfaces }\end{array}$ & $\begin{array}{l}\text { Statistical } \\
\text { Interfaces }\end{array}$ & $\begin{array}{c}\text { Filtered \& Statistical } \\
\text { Interfaces }\end{array}$ & $\begin{array}{l}\text { Statistical } \\
\text { Interfaces }\end{array}$ \\
\hline $\begin{array}{c}\text { Nb of fields } \\
1-F \\
2-F \\
\text { n-F } \\
\end{array}$ & $1-\mathrm{F}$ & $1-\mathrm{F}$ & $\begin{array}{l}1-\mathrm{F} \\
2-\mathrm{F} \\
\mathrm{n}-\mathrm{F}\end{array}$ & $\begin{array}{l}1-\mathrm{F} \\
2-\mathrm{F} \\
\mathrm{n}-\mathrm{F}\end{array}$ & $\begin{array}{l}1-\mathrm{F} \\
2-\mathrm{F} \\
\mathrm{n}-\mathrm{F}\end{array}$ & $\begin{array}{l}1-\mathrm{F} \\
2-\mathrm{F} \\
\mathrm{n}-\mathrm{F}\end{array}$ \\
\hline Two-phase models & $\begin{array}{l}\text { Pseudo } \\
\text { DNS }\end{array}$ & $\begin{array}{l}\text { LES with } \\
\text { simulated } \\
\text { interfaces }\end{array}$ & $\begin{array}{c}\text { Hybrid } \\
\text { LES with } \\
\text { filtered } \\
\text { \& statistical } \\
\text { interfaces }\end{array}$ & $\begin{array}{c}\text { LES } \\
\text { with } \\
\text { statistical } \\
\text { interfaces }\end{array}$ & $\begin{array}{c}\text { RANS } \\
\text { URANS } \\
\text { with filtered } \\
\text { \& statistical } \\
\text { interfaces }\end{array}$ & $\begin{array}{l}\text { Porous medium approach } \\
\text { statistical interfaces }\end{array}$ \\
\hline
\end{tabular}


CFD and two-phase CFD is in the domain of space filtered approaches. Three different approaches characterized by three different treatments of the interfaces correspond to the unique LES approach for single-phase CFD.

System codes use space integration of equations:

- Integration over a volume for the OD models or lumped parameter models

- Integration over a cross section for the 1D models

- Integration over one space direction for the 2D models, such as 2D pressure vessel downcomer modeling where equations are integrated over the radial direction

\subsection{DNS, Pseudo-DNS, and LES with Simulated Interfaces}

No averaging or filtering is used in single-phase DNS, which predicts the smaller scale eddies up to the Kolmogorov dissipative scale. In two-phase flow, some additional equation or numerical treatment is required to track the interface and to add the physics of either the liquid-solid interface or the triple line (liquid-solid-gas). Additional models are required; for example, when implementing a film-splitting criterion when two bubbles coalesce or for predicting the contact angles at a triple line. Such additional models illustrate why in two-phase flow the term DNS must be replaced by pseudo-DNS, since some very small scale physics is merely modeled, rather than solved. Since pure DNS without any modeling does not exist in two-phase flow, pseudo-DNS techniques also have to be validated against experimental data.

Both DNS and pseudo-DNS require extremely expensive CPU cost and will be used for simulating very small scale flow processes as a complement to experimental investigations. They will help understanding the local flow phenomena and will be used for developing closure relations for more macroscopic models.

A potentially cost-efficient alternative to pseudo-DNS is LES with simulated interfaces, which may reduce the CPU cost by one or two orders of magnitude without degradation of the predictions. However, LES with simulated interfaces is still very expensive and is not expected to be used directly in industrial simulations, although there have been first applications of this method to derive models for more macroscopic models in bubbly flow $[27,28]$ and in stratified flow $[29,30]$.

\subsection{CFD in the RANS or URANS Approach}

The most advanced and the most commonly used available single-phase and two-phase CFD method is the RANS or URANS approach. The time averaging filters all (or most) of the turbulent scales and all of the twophase intermittency scales. The method is applied to steady flows or quasi-steady flows when the time scales of variation of mean variables are larger than the largest time scales of turbulence and two-phase intermittency. For dispersed bubbly flow or dispersed droplet flow, the condition is easy to satisfy. The presence of interfaces is treated statistically by averaged parameters, such as the void fraction or the interfacial area density. However, a "Filtered Interface" treatment is possible for large interfaces like the free surface in a stratified flow [31, 32] or the film interface in an annular flow. For slug and churn flow regimes with large bubbles (either Taylor bubbles in slug flows or distorted large bubbles), the intermittency due to the passage of these bubbles corresponds to relatively large time scales. Since the RANS filters even these large scales, it is not able to predict this intermittency.

In single-phase situations, RANS and URANS approaches have been applied to several mixing problems, either as a stand-alone simulation (e.g. for containment mixing of air, steam, and hydrogen) or even with coupled simulations between CFD and system codes developed for issues such as boron mixing, mixing of hot and cold water in Steam Line Break (SLB) accidents, and Pressurized Thermal Shock (PTS).

In two-phase flow conditions, RANS and URANS have been applied within the NURESIM project for boiling flow [17 to 26] and for two-phase PTS scenarios [33].

\subsection{The LES with Statistical inTerfaces}

When the largest two-phase scale is smaller than the largest turbulent scale, an LES method may be applied. In such condition, to allow for a statistical treatment of the interfaces, the filter scale should be smaller than the larger eddies, but larger than the two-phase scale. This technique, which has already been applied with some success to turbulent dispersed flow [34], clearly consumes much less CPU time than the pseudo-DNS and the LES with deterministic interface. However, such a method is only applicable for certain flow regimes, such as dispersed bubble or dispersed droplet flows where the large eddies are much larger than the largest bubbles or droplets. The LES with statistical interface may also be used in a multiscale approach to capture large scale flow fluctuations and/or to derive averaged models for interfacial forces and turbulence closure laws of the RANS approach.

\subsection{The Hybrid LES Method with Both Filtered and Statistical InTerfaces}

This filtered method may be applied when $\mathrm{l}_{2 \varphi}<\delta_{\mathrm{f}}<\mathrm{L}_{2 \varphi}$ some interfaces being filtered, the smallest being statistically treated.

In principle, this technique may address all flow regimes with a more reasonable CPU cost than the pseudo-DNS and the LES methods with simulated interfaces. Thus, the hybrid LES method may be a promising way of modeling the most complex two-phase flow (such as churn or slug flow) at a reasonable CPU cost without filtering the twophase structures as RANS does. However, the closure issue is rather complex and the present state of the art is not very well advanced for this method. 


\subsection{The CFD in Porous Medium}

The CFD in porous medium approach uses a space filtering of time-averaged basic equations multiplied by $\chi_{\mathrm{f}}$ or by the product $\chi_{\mathrm{k}} \cdot \chi_{\mathrm{f}}$, as defined above. This approach is adapted to the macroscopic 3D description of two-phase flow in reactor components such as the core or the steam generator. The minimum filter scale in such components is the sub-channel scale. The CFD in porous medium approach is used with some simplifying assumptions in the component codes for the core and SGs. Due to space filtering, dispersion terms of momentum and energy appear in the equations, which would require some modeling. In the present state of the art, no modeling of dispersion terms exists and only very simple turbulent diffusion models are used. This scale is used in component codes and in 3D modules of system codes, and will be used in a multi-scale approach as the end product receiving information from smaller scale CFD methods for open medium.

\subsection{Application of CFD Methods for Nuclear Reactor Safety}

The OECD/NEA/CSNI has promoted various activities with the hope of applying CFD to Nuclear Reactor Safety (NRS). Three writing groups were created under the auspices of the Working Group for the Analysis and Management of Accidents (WGAMA) to produce stateof-the-art reports on different aspects of the subject. The first group, WG1, established Best Practice Guidelines
(BPG) [35] for CFD application in the field of Nuclear Reactor Safety (NRS). The second group, WG2, documented the existing assessment databases [36] for CFD application to some identified NRS issues. The third group, WG3, established some requirements for extending CFD codes to two-phase flow safety problems and produced two reports $[37,38]$. In addition to these activities, three workshops on the application of CFD to NRS were organized in Garching (CFD4NRS, Germany, 2006), Grenoble (XCFD4NRS, France, 2008), and Washington D.C. (CFD4NRS-3, USA, 2010). The main objective of the OECD activities on CFD has been to create a common CFD culture between code developers of the R\&D community, code users from the industry, and safety evaluators. The BPGs defined by WG1 [35] provide evaluators with some guidance for measuring the reliability of a CFD application. The WG2 report serves to identify assessment requirements for CFD applications to safety issues. Both WG1 and WG2 reports mainly address single-phase CFD since this technology has already a reasonable degree of maturity.

The two-phase CFD is less mature, and the WG3 report $[37,38]$ only addressed some preliminary questions, calling for a classification of the various methods and proposing a multi-step methodology. The group also defined the state of the art for some applications and produced a first set of recommendations and guidelines.

Table 2 summarizes the applicability and degree of

Table 2. Applicability and Degree of Maturity of the Various Two-Phase CFD Approaches to Every Flow Regime Including CPU Cost

\begin{tabular}{|c|c|c|c|c|c|}
\hline & Pseudo-DNS & $\begin{array}{c}\text { LES } \\
\text { Simulated } \\
\text { interfaces }\end{array}$ & $\begin{array}{c}\text { LES } \\
\text { Statistical \& filtered } \\
\text { interfaces }\end{array}$ & $\begin{array}{c}\text { LES } \\
\text { Statistical } \\
\text { interfaces }\end{array}$ & $\begin{array}{c}\text { RANS-URANS } \\
\text { Statistical \& filtered } \\
\text { interfaces }\end{array}$ \\
\hline Bubbly & $\mathrm{OK}$ & OK & $\mathrm{OK}$ & OK & $\mathrm{OK}$ \\
\hline Slug-Churn & $\begin{array}{c}\text { OK } \\
\text { Too expensive }\end{array}$ & $\begin{array}{c}\text { OK } \\
\text { Too expensive }\end{array}$ & Possible & No & possible \\
\hline Annular & $\begin{array}{c}\text { OK } \\
\text { Expensive }\end{array}$ & $\begin{array}{c}\text { OK } \\
\text { Expensive }\end{array}$ & Possible & No & $\mathrm{OK}$ \\
\hline Annular-mist & $\begin{array}{c}\text { OK } \\
\text { Too expensive }\end{array}$ & $\begin{array}{c}\text { OK } \\
\text { Too expensive }\end{array}$ & Possible & No & possible \\
\hline Mist flow & $\mathrm{OK}$ & OK & $\mathrm{OK}$ & OK & $\mathrm{OK}$ \\
\hline Stratified & $\mathrm{OK}$ & $\mathrm{OK}$ & $\mathrm{OK}$ & No & $\mathrm{OK}$ \\
\hline Stratified-mist & $\begin{array}{c}\text { OK } \\
\text { Too expensive }\end{array}$ & $\begin{array}{c}\text { OK } \\
\text { Too expensive }\end{array}$ & Possible & No & Possible \\
\hline All flow regimes & Too expensive & Too expensive & Possible & No & Possible \\
\hline
\end{tabular}

OK: is applicable and has been applied

Possible: can be applied but not very mature

Too expensive: unaffordable with current computer power
No: cannot be applied due to intrinsic limitations

Expensive: requires a very high CPU time 
maturity of the various two-phase CFD approaches for every flow regime, showing that the RANS approach can address all flow regimes and has already some maturity for dispersed flow and separate-phase flow. In principle, the hybrid LES method can also address all flow regimes, though it still requires an important modeling effort. Pseudo-DNS and LES with simulated interfaces are still limited by very high CPU costs, and thus will not be used directly for solving reactor issues, though they will play a role in multi-scale analysis.

\section{EXAMPLES OF MULTI-SCALE ANALYSIS}

In the hopes of improving closure laws of the porous medium approach and of 0D, 1D and 2D models of system codes, the OECD-CSNI working groups identified several issues related to reactor safety as potential applications of CFD for open medium. A few examples are given below.

\subsection{Subcooled Boiling, and Boiling Up to DNB in a PWR}

CFD modeling of subcooled boiling allows a mechanistic modeling of the interfacial mass, momentum, and energy transfers for bubbly flow in a heated channel. The evaporation rate on the heated wall depends on the local heat flux, as well as on the local liquid velocity, temperature, and turbulence, all of which are predicted at the CFD scale. The local void fraction depends on condensation in the bulk flow, which depends on both the local temperature and the bubble diameter, which are also predicted at CFD scale. The void distribution depends on all interfacial forces, including drag, lift, turbulent dispersion, wall lubrication, and added mass, all of which are modeled at the CFD scale.

It is possible to produce a RANS modeling of boiling flow up to DNB occurrence. This method may account for the geometrical effects related to the spacer design and may predict the "non-uniform heat flux effect," which must be modeled at the sub-channel scale. But such a CFD approach requires the existence of a local DNB criterion, and in the present state of the art, no physically based local DNB criterion exists. A simple empirical model allows some CHF prediction, but the accuracy is not yet satisfactory (see [17 to 26]). However, the CFD simulations may still be used for parametric studies as a tool to help fuel design and to reduce the need of experiments.

Pseudo-DNS is a way to investigate the local process responsible for the DNB and to develop the required local DNB criterion. LES with simulated interfaces of a boiling bubbly flow may also be used to derive closure laws for turbulence equations, coalescence and break-up of bubbles, turbulent dispersion modeling, and averaged lift force modeling.

\subsection{Dry-Out Investigations in a BWR Core}

Dry-out, which is associated with evaporation and disappearance of the liquid film on heated walls, typically occurs in high quality flows at either slug or annular twophase flow regime. Most of the phenomenological dryout models for system codes or component codes are based on mass conservation in the liquid film. Such models account for droplet entrainment and deposition, as well as liquid film evaporation. The entrainment and deposition rates are very sensitive to the geometry of the fuel assembly and particularly to the spacer grids. CFD with Lagrangian Particle Tracking (LPT) of droplets may be used to model deposition $[39,40]$ and the effects of spacer grids.

\subsection{Steam Generator Flows and Tube Vibrations}

Two-phase flow in steam generators is simulated by current component codes with a CFD for porous body approach. These component codes are used for predicting the global efficiency of the heat exchange as a function of the design characteristics, as well as to investigate fouling, corrosion, and tube vibration. The predicted flow parameters, such as velocity and steam quality, are averaged at a scale larger than the sub-channel. CFD in open medium may be beneficial in allowing for the investigation of flow processes at a smaller scale. Such a technique should produce better predictions of particle deposition with local effects due to the geometry. The prediction of density and velocity fluctuations of the twophase flow may also provide some spectral information for the fluid-structure interaction.

\subsection{Core Reflooding}

CFD simulations of the flow with superheated steam and droplets in the dry zone of the core during a reflooding process may bring additional information which is currently not accessible by available measuring techniques. CFD may predict the transverse profiles of velocity, temperature, and void fraction, and these figures can then be used to evaluate the error made by simplifications and assumptions of current models of system codes. The steam-to-droplet heat flux plays a very important role in the process, by transporting the heat received from the rods to the droplets. A classical model is:

$$
\begin{aligned}
& q_{v i}=\frac{6(1-\alpha)}{\delta} \frac{N u \lambda_{v}}{\delta}\left(T_{\text {sat }}-T_{v}\right) \\
& N u=2+0.57 \operatorname{Re} v^{0.5} \operatorname{Pr}^{0.33}
\end{aligned}
$$

Present system codes do not model profile effects, even though the difference between the space-averaged function $\langle q v i(X j)\rangle$ which is to be modeled and the function of the space-averaged flow parameters $q v i(\langle X j\rangle)$ which is used in the closure law can often be quite large. CFD may allow for the development of a model which can account for these profile effects.

CFD simulations may also determine the influence of the droplets on the wall-to-steam convective heat transfer coefficient. 
CFD simulations associated with Lagrangian Particle Tracking (LPT) of droplets may also bring information on the frequency of non-rewetting drop impact on the walls. If experimental data or pseudo-DNS simulations provide the power exchange for every non-rewetting drop impact, a model may be derived for the dispersed flow film-boiling heat transfer.

Pseudo-DNS or LES with simulated interfaces can also be used to develop physically based models for the following processes:

- Creation of droplets by film splitting at a quench front, with prediction of the drop size

- Droplet splitting by a non-rewetted spacer grid

- Droplet entrainment from liquid films along rewetted spacer grids, with prediction of the drop size

\subsection{Reflooding of a Debris Bed}

In case of a severe accident, a debris bed may be cooled by ECCS water. The reflooding of this debris bed must be modeled at the porous body scale for severe accidents codes. RANS-CFD simulations, or even pseudo-DNS used at the scale of the cell of the debris bed, may be used to develop the models for the 3D model for porous body.

\subsection{Local Effects in Spacer Grids or Support Plates}

Spacer grids or mixing grids in PWR or BWR rod assemblies play an important role in the efficiency of the fuel cooling in the mixing between sub-channels. These grids affect the value of the CHF as it relates either to the DNB or Dry-out. CFD simulations of the flow through these grids using either RANS or filtered approaches may be used to determine several factors related to the grids including the mixing between sub-channels, the pressure drop, the additional turbulence intensity, and the enhanced fluid-to-rod heat transfer coefficient. Spacer grids in PWR assemblies also affect bubble migration and dispersion, which consequently affect DNB occurrence. Spacer grids in BWR assemblies can affect droplet deposition and entrainment, which affects Dry-out occurrence. CFD simulations may replace costly experiments, or at least reduce the need of such experiments.

\subsection{Local Effects in Complex Geometries}

A reactor has many components with very complex geometries. Thus, classical thermalhydraulic models, which were developed from the analysis of experimental data obtained in established flow in simple geometry, are not directly applicable in such conditions. CFD simulations may help in identifying how classical models can be modified to take these local effects into account. Such investigations may be used to determine how bends, valves, nozzles, perforated plates, and diaphragms affect pressure losses, flow regime, slip ratio, and Counter Current Flow Limitation (CCFL). CFD simulations may also reveal form loss coefficients, flooding limit correlations, and local multipliers for interfacial friction, to be used in 1D models or 3D porous models of system codes.

Other reactor components like separators and dryers are modeled in system codes through empirical phase separation laws, which means that experimental tests are necessary for any change in the geometry. However, CFD simulations may at least be used for parametric studies in the design process to reduce the need of experimental testing.

\section{PERSPECTIVE FOR FUTURE APPLICATION OF A MULTI-SCALE ANALYSIS}

The continuous progress of computer power will increase the market share of CFD application in reactor thermalhydraulics, but this process will be rather slow. For the next few decades, macroscopic approaches using system and component codes will continue to play a dominant role for solving most LWR thermalhydraulic issues.

Every decade, computer power tends to increase by a factor of about 100. This is true for single processor computers as well as for massive, parallel High Power Computing (HPC). Considering the solution methods of 3-D thermalhydraulic equations, an increase in computer power by a factor of 100 should allow for an increase of a factor of about 15 on the number of meshes and a decrease of the mesh size by a factor of about 2.5 .

A rough estimation of the required number of meshes to simulate a single-core sub-channel thermalhydraulics in a typical boiling situation with a DNS approach is about $10^{15}$. This corresponds to approximately $10^{20}$ meshes for the whole core.

A similar estimation for a two-phase CFD for open medium approach using a RANS model gives approximately $10^{6}$ meshes for a single-core sub-channel and $10^{11}$ meshes for the whole core. This shows that two-phase CFD for open medium almost certainly will not replace component and system TH codes in the short and medium term (the next two decades). However, two-phase CFD for open medium may be used for two kinds of applications:

- Local zoom on a reactor component or part of a component during a part of a reactor transient

- Physical analysis of a local process in order to understand phenomena and to develop better models for more macroscopic approaches

As of 2010, it takes a few CPU hours for a system code user to simulate a typical accidental transient by using a coarse 3-D Pressure Vessel modeling (e.g. 1000 meshes) with a single processor computer. This coarse nodalization corresponds to an average mesh size of about $0.4 \mathrm{~m}$. In 2030, the same simulation with a single processor computer using the same CPU time would allow for a mesh refinement up to about $6 \mathrm{~cm}$. This simple estimation shows that the impressive progress of computer power in 
the next few decades still will (most likely) not allow for the transition from a porous body approach to a CFD for open medium for current applications. Moreover, the use of system codes for safety requires a Best-Estimate Plus Uncertainty (BEPU) approach. In the most widely used BEPU method, a Monte-Carlo type technique requires a rather large number of calculations of the same transient, with every uncertain parameter being randomly altered according to predetermined probability density functions. Therefore, the increase in computer power may first be used to multiply the number of calculations before reducing the mesh size. The trend towards "risk-informed regulations" may also lead to an increase in the number of simulations of accident scenarios.

A typical current application of a component code uses $10^{4}$ to $10^{6}$ meshes for a core with a porous body approach or in the context of a sub-channel analysis. In the case of a sub-channel analysis, only a few sub-channels or a single fuel assembly is modeled. A modeling of the whole core with a porous body approach does not allow for a converged meshing. Most applications combine a rather coarse nodalization of the entire core with a finer nodalization in the part of the core where attention is focused. The expected technological evolution of the next two decades should allow for a better space resolution of the porous body approach and/or a more extended use of sub-channel modeling.

Single-phase CFD application to some reactor issues is primarily used with RANS and URANS approaches, which require a more reasonable CPU time than LES or VLES approaches. However, looking at past international benchmarks, it is safe to say that the high CPU cost remains the main difficulty in obtaining a reliable simulation with a converged meshing. The strict application of Best Practice Guidelines is difficult when validation experiments are simulated and is even more critical for any reactor application. Therefore, it seems likely that future increases in computer power will be used first to acquire converged meshing, and then to extend the domain of application of CFD. The same conclusion applies to the few two-phase CFD applications.

The cost and availability of HPC for nuclear engineering and for the R\&D community will probably restrict the use of this technology to a few selected reactor issues for which it is necessary or brings a real added value. In the next two decades, HPC will likely be applied to issues such as:

- Safety issues with single-phase turbulent flow, such as boron mixing, mixing of hot and cold water during steam line break accidents, containment mixing of air steam and hydrogen, Pressurized Thermal Shock (PTS), and thermal stripping

- A more limited number of safety issues with twophase flow, such as some PTS scenarios

- Coupled problems such as TH-core physics and fluid-structure interaction

In addition to this direct application to reactor issues,
HPC may also play a role in basic research by providing "numerical experiments" or reference calculations in a multi-scale analysis approach, in examples such as:

- DNS or LES reference simulations of single-phase situations to evaluate the capability of RANS and URANS models to adequately capture the phenomena, and to measure the accuracy of RANSURANS predictions. Such simulations may be used either in the context of basic research or as a support to CFD application to safety demonstrations.

- Two-phase pseudo-DNS of boiling flow used as "numerical experiments" to investigate micro-scale flow processes which are not clearly visible by available experimental techniques, such as the DNB.

- Two-phase pseudo-DNS "numerical experiments" of prototypical flow configuration to derive averaged models for CFD in porous medium, CFD in open medium, or even 1D model of system codes.

\section{DIRECTIONS FOR FUTURE RESEARCH AND DEVELOPMENT}

Reactor thermalhydraulics will use several simulation tools listed above to solve all design and safety issues. In two-phase conditions, all modeling approaches need some validation and specific experimental programs, as well as advanced measurement techniques. The further development of local measurements techniques is mandatory to obtain reliable and validated physical models for various CFD approaches. The progress of two-phase CFD is currently hindered by a lack of experimental information. Each application of each modeling approach should identify a validation program and build new specific experiments. The finest CFD approaches may need only rather simple experimental tests, and have demonstrated better capabilities for extrapolation to more complex industrial geometries.

In the future, more effort should be spent to develop and apply methods for deriving averaged information from microscopic simulations (DNS, pseudo-DNS, LES), in view of developing closure laws for averaged models.

More effort should also be spent to investigate numerical and physical aspects of the coupling between two scales, particularly in two-phase conditions.

Success in the multi-scale approach requires the participation of experts in each domain, from DNS tools to system codes, who must cooperate and communicate in order to establish the synergy needed to create a new expertise in "multi-scale analysis."

In order to establish the credibility of CFD with safety evaluators, more precise BPGs must be given, more specific validation and verification plans must be developed, and international benchmarks must be organized.

\section{ACKNOWLEDGEMENTS}

The author is grateful to the members of the NEPTUNE 
project for a multi-scale thermalhydraulic platform and to CEA, AREVA, EDF and IRSN, who financed the project. Thanks are extended to the members of the EUROFASTNET and ECORA projects (5th Framework program), the NURESIM project (6th Framework program), and the NURISP project (7th Framework program), who contributed to the multi-scale analysis, and to the European Commission who funded these projects.

\section{GLOSSARY}

$\begin{array}{ll}\text { BEPU } & \text { Best-Estimate Plus Uncertainty } \\ \text { BPG } & \text { Best Practice Guidelines } \\ \text { BWR } & \text { Boiling Water Reactor } \\ \text { CFD } & \text { Computational Fluid Dynamics } \\ \text { CHF } & \text { Critical Heat Flux } \\ \text { CSNI } & \begin{array}{l}\text { Committee on the Safety of Nuclear } \\ \end{array} \\ \text { Installations } \\ \text { DNB } & \text { Departure from Nucleate Boiling } \\ \text { DNS } & \text { Direct Numerical Simulation } \\ \text { ECCS } & \text { Emergency Core-Cooling System } \\ \text { ITM } & \text { Interface Tracking Method } \\ \text { LBLOCA } & \text { Large-Break Loss Of Coolant Accident } \\ \text { LES } & \text { Large Eddy Simulation } \\ \text { LOCA } & \text { Loss Of Coolant Accident } \\ \text { LPT } & \text { Lagrangian Particle Tracking } \\ \text { LWR } & \text { Light Water Reactor } \\ \text { NEA } & \text { Nuclear Energy Agency } \\ \text { NRS } & \text { Nuclear Reactor Safety } \\ \text { OECD } & \text { Organization for Economic Cooperation } \\ & \text { and Development } \\ \text { PTS } & \text { Pressurized Thermal Shock } \\ \text { PWR } & \text { Pressurized Water Reactor } \\ \text { RANS } & \text { Reynolds-Averaged Navier-Stokes } \\ \text { TH } & \text { thermalhydraulic } \\ \text { URANS } & \text { Unsteady Reynolds-Averaged Navier- } \\ & \text { Stokes } \\ \text { VLES } & \text { Very Large Eddy Simulation } \\ \text { VOF } & \text { Volume-Of-Fluid } \\ \text { WGAMA } & \text { Working Group on the Analysis and } \\ & \text { Management of Accidents } \\ & \end{array}$

\section{REFERENCES}

[1] M. Robert, M. Farvacque, M. Parent, and B. Faydide, "CATHARE 2 V2.5: A Fully Validated CATHARE Version for Various Applications," Proc. 10th Int. Topl. Mtg. Nuclear Reactor Thermal Hydraulics (NURETH 10), Seoul, Republic of Korea, October 5-9, 2003.

[2] P. Emonot, A. Souyri, J.L. Gandrille, F. Barré, CATHARE 3: A new system code for thermal-hydraulic in the context of the NEPTUNE project, NURETH-13, Kanazawa City, Ishikawa Prefecture, Japan, September 27-October 2, 2009

[ 3 ] RELAP5/MOD3 Code Manual, Volume I: Code Structure, System Models and Solution Methods, NUREG/CR-5535, 1995

[4] TRACE-V5.0, Theory Manual, 2007, TRACE V5.0 User Manual, 2007, TRACE V5.0 Assessment Manual, 2007
[5] M.J. Burwell, D. Enix, E. Lerchl, J. Miro, V. Teschendorff, and K. Wolfert, The Thermal-Hydraulic Code ATHLET for Analysis of PWR and BWR Systems, Fourth Int. Meeting on Nuclear Reactor Thermal-Hydraulics, NURETH-4, Proceedings Vol. 2, pp. 1234-1239, 1988

[6] Proceedings of OECD/CSNI Specialist Meeting on Transient Two-Phase Flow - System Thermalhydraulics, Aix-EnProvence (F), 1992

[ 7 ] Proceedings of OECD/CSNI Workshop on Transient Thermal-Hydraulic and Neutronic Codes Requirements, 5-8 November 1996, Annapolis, Md, USA, NUREG/CP-0159, NEA/CSNI/R(97)4

[ 8 ] Proceedings of "OECD/CSNI Workshop on Advanced Thermal-Hydraulic and Neutronic Codes: Current and Future Applications", Barcelona (E), 2000

[9] D. Bestion, P. Clément, J.P. Caminade, J.M. Delhaye, P. Dumaz, J. Garnier, D. Grand, E. Hervieu, O. Lebaigue, H. Lemonnier, C. Lhuillier, J.R. Pages, I. Toumi, M. Villand, FASTNET, A proposal for a ten-year effort in ThermalHydraulic research, Multiphase Science \& Technology, Vol. 11, pp. 79-145, 1999, Editors: J.M. Delhaye, J. Garnier

[10] D. Bestion, A. Latrobe, H. Paillère, A. Laporta, V. Teschendorff, H. Staedtke, N. Aksan, F. d'Auria, J. Vihavainen, P. Meloni, G. Hewitt, J. Lillington, B. Mavko, A. Prosek, J. Macek, M. Malacka, F. Camous, F. Fichot, and D. Monhardt, "European Project for Future Advances in Science and Technology for Nuclear Engineering Thermal-Hydraulics - EUROFASTNET, Final Report," Technical Report, Commission of the European Communities, 2002.

[11] D. Bestion, A. Guelfi, « Status and perspective of twophase flow modelling in the NEPTUNE Multiscale thermalhydraulic platform for nuclear reactor simulation », Nuclear Engineering and Technology, 2005, Vol. 16, Nos 1-3, pp 1-5.

[12] A.Guelfi, D. Bestion, M. Boucker, P. Boudier, P. Fillion, M. Grandotto, J.M. Herrard, E. Hervieu, P. Peturaud, NEPTUNE A new Software Platform for advanced Reactor Thermalhydraulics, Nuclear Science and Engineering, 156, 282-324, 2007

[13] C. Chauliac, J.M. Aragonés, D. Bestion, D.G. Cacuci, N. Crouzet, F.P. Weiss, M. A. Zimmermann , NURESIM A European simulation platform for nuclear reactor safety: multi-scale and multi-physics calculations, sensitivity and uncertainty analysis, FISA 2009, Praha, Czech Rep., June 22-24, 2009

[14] D. Bestion, D. Lucas, B. Smith, M. Boucker, M. Scheuerer, F. d'Auria, D. Lakehal, J. Macek, I. Tiselj, G. Hazi, V. Tanskanen, M. Ilvonen, N. Seiler, M. Boetcher, H. Anglart, Y. Bartosiewicz, Two-phase CFD advances in the NURESIM and NURISP projects , 18th International Conference on Nuclear Engineering ICONE 18, May 1721, 2010, Xi'an, CHINA

[15] D. Bestion, Extension of CFD Code application to TwoPhase Flow Safety Problems, Nuclear Engineering and Technology, VOL.42, No.4, August 2010

[16] D. Bestion, Applicability of two-phase CFD to nuclear reactor thermalhydraulics and elaboration of Best Practice Guidelines, CFD4NRS-3, Washington-DC, sept. 2010

[17] NURESIM D2.2.1: Review of the existing data basis for the validation of CFD models for CHF, www.nuresim.com

[18] NURESIM D2.2.1.1c: Synthesis of the work performed in 
WP2.2 on CHF investigations using two-phase CFD, www.nuresim.com

[19] C. Morel, Wei Yao, D. Bestion, Three Dimensional modelling of boiling flow, The 10th International Topical Meeting on Nuclear Reactor Thermal Hydraulics (NURETH-10), Seoul, Korea, October 5-9, 2003

[20] M. Boucker, A. Guelfi, S. Mimouni, P. Péturaud, D. Bestion, E. Hervieu, Towards the prediction of local thermalhydraulics in real PWR core conditions using NEPTUNE_CFD software, Workshop on Modeling and Measurements of Two-Phase Flows and Heat Transfer in Nuclear Fuel Assemblies, KTH, Stockholm, Sweden - 10-11 October 2006

[21] D. Lucas, E. Krepper, H.M. Prasser, Modelling of the evolution of large bubbly flow along a vertical pipe, 2007, Nuclear Technology 158, 291-303

[22] S. Mimouni, F. Archambeau, M. Boucker, J. Laviéville, CFD Modelling of subcooled boiling in the NEPTUNE_CFD code and application to fuel assembly analysis , XCFD4NRS, GRENOBLE, FRANCE, Sept10-12, 2008

[23] J. Macek, L. Vyskocil, Simulation of Critical Heat Flux Experiments in NEPTUNE_CFD Code XCFD4NRS, Grenoble, France, 10 - 12 September 2008

[24] D. Bestion, H. Anglart, D. Caraghiaur, P. Péturaud, B. Smith, M. Andreani, B. Niceno, E. Krepper, D. Lucas, F. Moretti, M. C. Galassi, J. Macek, L. Vyskocil, B. Koncar, and G. Hazi, Review of Available Data for Validation of Nuresim Two-Phase CFD Software Applied to CHF Investigations, Science and Technology of Nuclear Installations, Volume 2009 (2009), Article ID 214512

[25] C. Morel and J. M. Laviéville, Modeling of Multisize Bubbly Flow and Application to the Simulation of Boiling Flows with the Neptune CFD Code, Science and Technology of Nuclear Installations, Volume 2009, Article ID 953527

[26] B. Koncar, E. Krepper, CFD simulation of convective flow boiling of refrigerant in a vertical annulus, Nucl. Eng. Des., Volume 238, Issue 3, 2008, pp. 693-706

[27] G. Bois, D. Jamet \& O. Lebaigue, 2010, "Towards Large Eddy Simulation of two-phase flow with phase-change: Direct Numerical Simulation of a pseudo-turbulent twophase condensing flow", 7th International Conference on Multiphase Flow, ICMF 2010, Tampa, FL, USA, May 30 - June 4, 2010

[28] S. Magdeleine, B. Mathieu, O. Lebaigue \& C. Morel, 2010, "DNS up-scaling applied to volumetric interfacial area transport equation, $12 \mathrm{p}$., 7th International Conference on Multiphase Flow", ICMF 2010, Tampa, FL, USA, May 30 - June 4, 2010.

[29] D. Lakehal: LEIS for the Prediction of Turbulent Multifluid
Flows Applied to Thermal Hydraulics Applications. XCFD4NRS, Grenoble, Sep. 10-12, 2008

[30] Lakehal D., Fulgosi M., Banerjee S., Yadigaroglu G.,: Turbulence and heat transfer in condensing vapor-liquid flow, Phys. Fluids, 20, 065101, 2008.

[31] Coste P., Pouvreau J., Morel C., Laviéville J., Boucker M., Martin A., "Modeling Turbulence and Friction around a Large Interface in a Three-Dimension TwoVelocity Eule-rian Code", Proc. of Int. Conf. NURETH 12, Pittsburgh, USA, 30 Septembre- 4 Octobre 2007.

[32] P. Coste, J. Laviéville, J. Pouvreau, C. Baudry, M. Guingo, A. Douce, "Validation of the Large Interface Method of NEPTUNE_CFD 1.0.8 for Pressurized Thermal Shock (PTS) Applications", Proc. XCFD4NRS, Washington DC, USA, 14-16 September 2010.

[33] D. Lucas, D. Bestion, E. Bodèle, P. Coste, M. Scheuerer, F. D'Auria, D. Mazzini, B. Smith, I. Tiselj, A. Martin, D. Lakehal, J.-M. Seynhaeve, R. Kyrki-Rajamäki, M. Ilvonen, and J. Macek, An Overview of the Pressurized Thermal Shock Issue in the Context of the NURESIM Project, Science and Technology of Nuclear Installations, Volume 2009 (2009), Article ID 583259

[34] B. Ničeno, M. Boucker, and B. L. Smith, Euler-Euler Large Eddy Simulation of a Square Cross-Sectional Bubble Column Using the Neptune_CFD Code, Science and Technology of Nuclear Installations, Volume 2009 (2009), Article ID 410272

[35] Mahaffy, J. (ed.) "Best Practice Guidelines for the Use of CFD in Nuclear Reactor Safety Applications", OECD, Nuclear Energy Agency, Technical Report, NEA/ CSN I /R(2007)5, April 2007

[36] Smith, B.L. (ed.), "Assessment of Computational Fluid Dynamics (CFD) for Nuclear Reactor Safety Problems", OECD Nuclear Energy Agency, Technical Report, NEA/CSNI/R(2007)13, Jan. 2008.

[37] D. Bestion, (ed.), Extension of CFD Codes to Two-Phase Flow Safety Problems, NEA/SEN/SIN/AMA(2006)2

[38] D. Bestion, H. Anglart, J. Mahaffy, D. Lucas, C.H. Song, M. Scheuerer, G. Zigh, M. Andreani, F. Kasahara, M. Heitsch, E. Komen, F. Moretti, T. Morii, P. Mühlbauer, B.L. Smith, T. Watanabe, Extension of CFD Codes to Two-Phase Flow Safety Problems, NEA-CSNI-R(2010)2

[39] D. Caraghiaur and H. Anglart, Annular flow deposition model with obstacle effect, 6th Int. Conf. on multiphase Flow ICMF, 2007, Leipzig,, Germany, July 9-13

[40] D. Caraghiaur and H. Anglart, Lagrangian particle tracking as a tool for deposition modelling in annular flow, ICONE-17, july 12-17, 2009, Brussels, Belgium 